\title{
Identification of catalytically important residues of the carotenoid 1,2-hydratases from Rubrivivax gelatinosus and Thiocapsa roseopersicina
}

\author{
Aida Hiseni ${ }^{1}$ - Linda G. Otten ${ }^{1}$ - Isabel W. C. E. Arends ${ }^{1}$
}

Received: 1 May 2015 /Revised: 1 September 2015 / Accepted: 10 September 2015 /Published online: 19 October 2015

(C) The Author(s) 2015. This article is published with open access at Springerlink.com

\begin{abstract}
Carotenoid 1,2-hydratases (CrtC) catalyze the selective addition of water to an isolated carbon-carbon double bond. Although their involvement in the carotenoid biosynthetic pathway is well understood, little is known about the mechanism by which these hydratases transform carotenoids such as lycopene into the corresponding hydroxyl compounds. Key residues were identified at positions His239, Trp241, Tyr266, and Asp268 in CrtC from Rubrivivax gelatinosus (and corresponding positions in Thiocapsa roseopersicina). Alanine mutants at these positions were found to be completely inactive, suggesting their direct involvement in the catalytic reaction. Our resulting mechanistic hypothesis is in analogy with the recently studied class of terpenoid cyclase enzymes containing a highly acidic aspartic residue in their active site. We propose that a similar aspartic acid residue, which is conserved through all putative CrtCs, is involved in initial protonation of the double bond in lycopene.
\end{abstract}

Keywords Carotenoid 1,2-hydratase $\cdot \mathrm{CrtC} \cdot$ Hydro-lyase $\cdot$ Enzyme mechanism $\cdot$ Biocatalysis

Electronic supplementary material The online version of this article (doi:10.1007/s00253-015-6998-y) contains supplementary material, which is available to authorized users.

Isabel W. C. E. Arends

I.W.C.E.Arends@tudelft.nl; http://www.bt.tudelft.nl/boc

1 Biocatalysis Group, Department of Biotechnology, Delft University of Technology, Julianalaan 67, 2628 BC Delft, The Netherlands

\section{Introduction}

Carotenoids, which represent one of the most abundant natural pigments with structural and protective properties (Armstrong and Hearst 1996), play an essential role in the photosynthetic machinery of phototrophic organisms such as purple bacteria (Jensen et al. 1961) and higher plants (Cazzonelli 2011). In addition, they have been identified in fungi and some nonphotosynthetic bacteria (Armstrong 1997). Carotenoid 1,2hydratase (also known as $\mathrm{CrtC}$ ) is a member of hydro-lyase group EC 4.2.1.131 (Hiseni et al. 2015). The enzyme takes part in the biosynthetic pathway of carotenoids (Umeno et al. 2005). CrtC introduces a tertiary hydroxyl group into an acyclic carotenoid molecule by addition of water to the carboncarbon double bond at the $\mathrm{C}-1$ position. The enzyme belongs to Pfam family PF07143 that encompasses members from several purple photosynthetic bacteria. On the other hand, CrtCs have been identified, which are able to hydrate monocyclic carotenoid gamma-carotene. These are evolutionary very distinct from the PF07143 members and have been given the name CruF (Sun et al. 2009).

To date, two representatives of the PF07143 family, the CrtCs from purple non-sulfur Betaproteobacteria Rubrivivax gelatinosus and purple sulfur Gammaproteobacteria Thiocapsa roseopersicina, were recombinantly expressed and characterized (Hiseni et al. 2011). Biochemical studies have revealed that these enzymes are able to convert cofactor-independently lycopene into 1-HO-lycopene and 1,1'-(HO) ${ }_{2}$-lycopene (Fig. 1). In addition, they showed some activity towards the unnatural substrate geranylgeraniol, a C20 molecule that resembles the natural substrate lycopene.

CrtCs are appealing enzymes in the biotechnology field because they are able to generate a tertiary alcohol, a highly valuable building block for the synthesis of several bioactive natural products and pharmaceuticals (Hiseni et al. 2015; Kourist et al. 


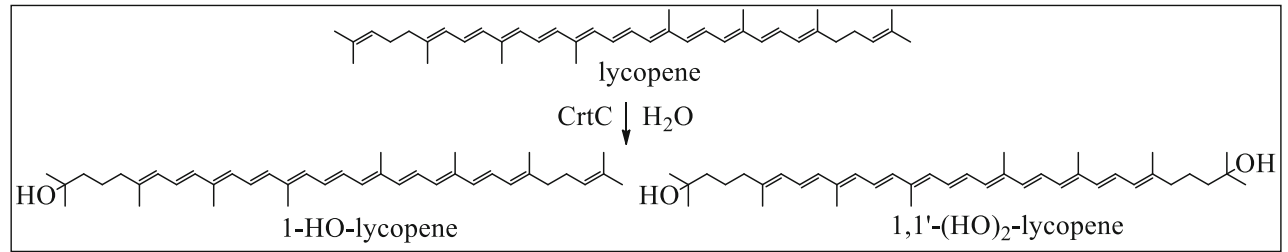

Fig. 1 Reaction catalyzed by Rubrivivax gelatinosus and Thiocapsa roseopersicina carotenoid 1,2-hydratase; the conversion of lycopene into 1-HOlycopene and 1,1'-(HO) $)_{2}$-lycopene

2008). Furthermore, they possess an intrinsically high stability at a wide $\mathrm{pH}$ and temperature range, which constitute useful properties for an industrial application (Hiseni et al. 2011). The subcellular location of this enzyme in the cell membrane fraction (membrane associated) allows for a straightforward isolation and simplified large-scale purification.

From a chemical point of view, CrtCs are able to perform a challenging chemical reaction, namely the selective addition of water to an isolated carbon-carbon double bond (Jin and Hanefeld 2011). Using the enzyme, the reaction proceeds without assistance of electron-withdrawing groups or transition metal cations, while the chemical hydration requires harsh acidic conditions (Evans and Kirby 1984). Furthermore, the CrtCs from photosynthetic bacteria act on acyclic carotenoids, whereas the CruFs from non-photosynthetic bacteria catalyze the hydration of monocyclic carotenoids. To our knowledge, no published data exist on the catalytic and structural features that determine hydratase activity and specificity of these two distinct families, nor has the 3D structure been elucidated yet. The mechanism of lycopene hydration, which involves proton attack at $\mathrm{C}-2$ and $\mathrm{C}-2^{\prime}$ and the introduction of the hydroxyl group at $\mathrm{C}-1$ and $\mathrm{C}-1^{\prime}$, was established from ${ }^{2} \mathrm{H}_{2} \mathrm{O}$ and $\mathrm{H}_{2}{ }^{18} \mathrm{O}$ labeling studies with intact cells (Patel et al. 1983; Yeliseev and Kaplan 1997). For a hydration reaction, it is likely to assume that the first step in the reaction is protonation of the alkene, leading to an intermediate carbocation. Quenching of the carbocation by water will lead to the alcohol as product. The protonation of hydrophobic long-chain alkenes has also been described for the enzyme class of cyclases, of which the full mechanism is known (Hammer et al. 2013; Wendt et al. 2000).

The objective of this study was to provide insight into the hydration mechanism of CrtCs. This knowledge is pivotal in order to engineer this promising enzyme class towards, i.e., higher activities, better stability, or widening of its substrate scope. Through multi-sequence alignment of several $\mathrm{CrtC}$ homologues, highly conserved amino acids were identified, which could be functionally or structurally important. The corresponding alanine mutants of these amino acids were produced to evaluate their involvement in the hydratase activity. Following the identification of probable catalytically active amino acid residues, the aim was to propose a catalytic mechanism for addition of water catalyzed by $\mathrm{CrtC}$.

\section{Materials and methods}

In silico analysis Basic Local Alignment Search Tool (BLAST) (Altschul et al. 1990) was used to find and select carotenoid 1,2-hydratase homologues of $\mathrm{CrtC}$ from $R$. gelatinosus ( $\mathrm{RgCrtC}$ ) using default settings; e.g., for nucleotide BLAST, the tblastn option was used with the nucleotide database (nt/nr), and for protein BLAST, the blastp option was used with the non-redundant protein database (nr). In order to look for identities/similarities between the $\mathrm{CrtC}$ homologues, nucleotide and amino acid sequences were aligned with the BioEdit Sequence Alignment Editor v.7.1.3.0 (www.mbio.ncsu.edu/bioedit/bioedit.html) or ClustalW (Larkin et al. 2007). In addition, protein sequences were subjected to protein functional analysis using a search in the Conserved Domain Database (CDD) (Marchler-Bauer et al. 2011) and Pfam search (Finn et al. 2010), using the standard parameters on the respective websites of these tools. A protein phylogenetic tree was constructed with Phylogeny. fr using the "One Click" program settings (Dereeper et al. 2010; Dereeper et al. 2008). These settings represent a default mode which proposes a pipeline already set up to run and connect programs recognized for their accuracy and speed to reconstruct a robust phylogenetic tree from a set of sequences (MUSCLE for multiple alignment, optionally Gblocks for alignment curation, PhyML for phylogeny, and finally, TreeDyn for tree drawing).

Cloning of carotenoid 1,2-hydratase genes Plasmids pET15b_CrtC $\mathrm{Rg}_{\mathrm{Rg}}$ and $\mathrm{pET} 15 \mathrm{~b} \_\mathrm{CrtC}_{\mathrm{Tr}}$ containing CrtC from $R$. gelatinosus $(R g)$ and T. roseopersicina $(T r)$, respectively, were constructed in a previous study (Hiseni et al. 2011). Two fosmids with $\operatorname{crtC}$ genes from metagenomic samples DelRiverFos06H03 (Fos06) and DelRiverFos13D03 (Fos13), respectively, were kindly provided by Dr. Kirchman (Waidner and Kirchman 2005). The cosmid encoding CrtC from Bradyrhizobium ( $\mathrm{Br}$ ) was received from Dr. Dreyfus (Giraud et al. 2004). In order to get sufficient DNA material for further studies, the fosmid DNA and cosmid DNA were amplified in Escherichia coli TOP10 cells. After DNA isolation with the QIAprep Spin Miniprep Kit (Qiagen) from the cells, sufficient DNA was obtained for further research. The crtCs from Rhodospirillum rubrum $(R r)$ and Rhodopseudomonas palustris $(R p)$ were amplified from 
genomic DNA. For that, genomic DNA of $R$. rubrum $(R r)$ was kindly provided by Prof. Roberts (NCBI Reference Sequence: NC_007641.1). R. palustris cells (DSM No. 123) were obtained from DSMZ (Deutsche Sammlung von Mikroorganismen und Zellkulturen), enriched in appropriate medium according to DSMZ instructions and gDNA isolated using the UltraClean Soil DNA Isolation Kit (Mo Bio). Subsequently, primers were designed for the isolation of all $\mathrm{crtC}$ genes (Table 1), which carry two restriction sites for subsequent cloning: NdeI (forward) and XhoI (reverse). For BrcrtC, the $X h o I$ site was replaced with $B a m H I$, because the $X h o I$ site was present in the gene itself. Amplification reactions were done using standard PCR reactions. Using the appropriate enzymes, the fragment was digested, purified, and ligated into the same sites of the $\mathrm{pET} 15 \mathrm{~b}$ vector and transformed into $E$. coli TOP 10 competent cells. The insertion of the $\mathrm{crt} C$ gene was verified by restriction analysis with the corresponding restriction enzymes (New England Biolabs) and DNA sequencing (BaseClear, Leiden, The Netherlands).

Single point mutations Single amino acid exchange within the $\operatorname{crt} C$ genes of $R g$ and $\operatorname{Tr}$ was done using the megaprimer PCR method introduced by Kammann et al. (1989) and later modified by Sarkar and Sommer (1990) and Landt et al. (1990). The mismatch primers are listed in Table 2. In the first PCR reaction, performed under standard reaction conditions, the megaprimer was produced using the corresponding forward primer containing the desired base substitution (Table 2) in combination with the reverse primer Rg_rv or Tr_rv (Hiseni et al. 2011). Plasmids pET15b_CrtC $\mathrm{Rg}_{\mathrm{Rg}}$ and pET15b_CrtC ${ }_{\mathrm{Tr}}$ (Hiseni et al. 2011) were used as the template. The size and purity of the megaprimer was verified by agarose gel electrophoresis. In order to produce the full-length gene, a second PCR reaction was performed with the corresponding megaprimer and $R g$ fw or $T r$ fw (Hiseni et al. 2011). Subsequent steps were performed as described in the previous section. The presence of the desired mutation was verified by DNA sequencing (BaseClear, Leiden, The Netherlands).
N-terminally truncated $\mathrm{RgCrtC}$ and $\operatorname{Tr} \mathrm{CrtC} \mathrm{RgCrtC}$ and $\operatorname{Tr} \mathrm{CrtC}$ lacking the first 45 and 57 amino acids, respectively, were constructed using primers $\operatorname{Rg} 45 \mathrm{aa} / \operatorname{Rg}$ rv and $\operatorname{Tr} 57 \mathrm{aa} /$ Tr rv (Table 3; Hiseni et al. 2011) under standard PCR conditions. Plasmids pET15b_CrtC $\mathrm{Rg}_{\mathrm{Rg}}$ and $\mathrm{pET} 15 \mathrm{~b} \_\mathrm{CrtC}_{\mathrm{Tr}}$ (Hiseni et al. 2011), respectively, were used as the template. Subsequent steps including verification were performed as described in the "Cloning of carotenoid 1,2-hydratase genes" section.

Recombinant expression of CrtCs E. coli BL21 (DE3) was the host for the pET15_CrtC plasmids. Cultures were grown at $37^{\circ} \mathrm{C}$ in Luria-Bertani broth with $100 \mu \mathrm{g} \mathrm{ml}^{-1}$ ampicillin until an OD600 value of 0.6-0.8 was reached. Unless otherwise stated, protein expression was induced by addition of isopropyl- $\beta$-D-thiogalactopyranoside (IPTG) to a final concentration of $0.1 \mathrm{mM}$, followed by cultivation at $25^{\circ} \mathrm{C}$ overnight. The cells were harvested by centrifugation at 10 , $000 \mathrm{rpm}$ for $10 \mathrm{~min}$ at $4^{\circ} \mathrm{C}$ (Sorvall), washed once with $50 \mathrm{mM} \mathrm{Na}_{2} \mathrm{HPO}_{4}$ buffer ( $\mathrm{pH} \mathrm{8.0)}$, and suspended in the same buffer. In case of subsequent purification, $10 \mathrm{mM}$ imidazole was added to the buffer. Crude extract (CE) from cultures $>100 \mathrm{ml}$ was prepared by adding $1 \mathrm{mg} \mathrm{ml}^{-1}$ lysozyme and incubating the cells for $1 \mathrm{~h}$ at $4{ }^{\circ} \mathrm{C}$, followed by cell disruption at the pressure of $1.5 \mathrm{kBar}$ (Constant Systems; IUL Instruments). For cultures $<100 \mathrm{ml}$, the cells were disrupted by sonication for $2 \mathrm{~min}$ while immersed in an ice-water bath using the microtip probe of a sonicator (Branson Sonicator Cell Disruptor) set at $50 \%$ maximal energy. In an effort to reduce the liquid viscosity caused by DNA molecules, $0.1 \mathrm{mg} \mathrm{ml}^{-1}$ of DNAse was added. With the subsequent centrifugation at $10,000 \mathrm{rpm}$ for $20 \mathrm{~min}$ at $4{ }^{\circ} \mathrm{C}$, cell-free extract (CFE) and pellet were separated. Protein content of the crude extract was determined by BCA assay (Pierce) with bovine serum albumin as the reference protein.

CrtC purification $\mathrm{RgCrtC}$ and $\mathrm{Tr} \mathrm{CrtC}$ "active site" point mutants were purified from the membrane fraction,
Table 1 Primers used for PCR and subsequent cloning of the genes into the expression vector pET15b

\begin{tabular}{lll}
\hline Name & Sequence $\left(5^{\prime} \rightarrow 3^{\prime}\right)$ & Restriction site \\
\hline DRF06 FW & GGGAGTACCATATGAGTGATGATGGCCAAC & NdeI \\
DRF06 RV & ATCCGCTCGAGATAATCTCAAGCCCGCCTCG & XhoI \\
DRF13 FW & GGGAGTACATATGGATGGCGTGTCAGAC & NdeI \\
DRF13 RV & CCGCTCGAGTAATGCTTAGGGCCACTTGGC & XhoI \\
Br FW & CGGACATCATATGTGCCCGCCAG & NdeI \\
Br RV & ATCCAGGATCCATCGCGTGAACTTCACCACC & Bam $\mathrm{HI}$ \\
Rp FW & CGGGACTTCCATATGTCAGGAGCTGAGTTG & NdeI \\
Rp RV & ACCGCTCGAGTAACGTTCAGCGGAACGC & XhoI \\
Rr FW & GGGAATTCCATATGCACCGCCCGGAC & NdeI \\
Rr RV & GCTCGAGTTCAATTAGCCCTTAACCGCCGC & $X h o \mathrm{I}$ \\
\hline
\end{tabular}

The respective restriction sites are underlined 
Table 2 Primers for site-directed mutagenesis

\begin{tabular}{lll}
\hline & $\begin{array}{l}\text { Amino acid } \\
\text { exchange }\end{array}$ & Sequence $\left(5^{\prime} \rightarrow 3^{\prime}\right)$ \\
\hline R. gelatinosus & H239A & AGCGGCGGACGCGCTCGCTG \\
W241A & CATCGCGCGGGGCCGATCG \\
H264A & CTGGAGCGGCGCCGCCTACC \\
Y266A & GCCACGCCGCCCTCGACT \\
D268A & CGCCTACCTCGCCTCGAACGAAG \\
H237A & GATCCGGCGGACGCGCAGTCTGGTGG \\
W239A & CGCCATGTCGCGTGGCCGTC \\
H262A & GCTGGAGCGGCGCTGGCTAT \\
D266A & CATGGCTATCTCGCCTCAAA \\
S58V & GCGTCCGTCGTCGCGCAGCA \\
S58Q & GCGTCCCAGGTCGCGCAGCA \\
\hline
\end{tabular}

Mismatch points are underlined while the $T r$ "processing" mutants (S58V, S58Q) were purified from the CFE. The membrane fraction was obtained after the centrifugation of the CFE for $4 \mathrm{~h}$ at 13 , $200 \mathrm{rpm}$ and $8^{\circ} \mathrm{C}$. The membranes were homogenized by ca. 20 passages through a $25-\mathrm{G}$ needle. Ni-NTA Sepharose HP (GE Healthcare) (previously equilibrated in $50 \mathrm{mM} \mathrm{Na}_{2} \mathrm{HPO}_{4}$ buffer, $\mathrm{pH} 8.0,300 \mathrm{mM} \mathrm{NaCl}$, $10 \mathrm{mM}$ imidazole) was added to the CFE or membrane sample. The mixtures were incubated for $1 \mathrm{~h}$ at RT, loaded into a polypropylene tube with porous disc (GE Healthcare), and washed three times with washing buffer (50 $\mathrm{mM} \mathrm{Na}_{2} \mathrm{HPO}_{4}$ buffer, $\mathrm{pH} 8.0,300 \mathrm{mM} \mathrm{NaCl}$, $75 \mathrm{mM}$ imidazole). The $\mathrm{CrtC}$ protein was eluted from the column with elution buffer $\left(50 \mathrm{mM} \mathrm{Na} \mathrm{HPO}_{4}\right.$ buffer, pH 8.0, $300 \mathrm{mM} \mathrm{NaCl}, 1 \mathrm{M}$ imidazole). Enzyme fractions were separated by sodium dodecyl sulfatepolyacrylamide gel electrophoresis (SDS-PAGE) (10\% Bis-Tris; Bio-Rad) and visualized by staining with SimplyBlue SafeStain (Invitrogen).

Determination of enzyme activity Enzymatic activities were determined with $\mathrm{CE}$ using lycopene as the substrate according to the method described earlier (Hiseni et al. 2011), with few modifications. The assay was carried out with $50 \mu \mathrm{l} \mathrm{CE}$ and $20 \mu \mathrm{M}$ substrate, and $10 \mathrm{mg} \mathrm{ml}^{-1} \mathrm{~L}-\alpha$-phosphatidylcholine, in a reaction volume of $200 \mu$ l. Prior to the analysis, acetonitrile was added to the reaction mixture in a ratio of $60: 40(\mathrm{ACN} /$ $\mathrm{H}_{2} \mathrm{O}$ ), the mixtures were shaken vigorously for $1 \mathrm{~min}$, and

Table 3 Primers used for construction of truncated CrtCs

\begin{tabular}{ll}
\hline Name & Sequence $\left(5^{\prime} \rightarrow 3^{\prime}\right)$ \\
\hline Rg_45aa & AGTACCATATGGGCGACGCACGGCTGG \\
Tr_57aa & AGTACCATATGTCCGTCGCGCAGCAAGG
\end{tabular}

The NdeI restriction sites are underlined solids were removed by centrifugation for $1 \mathrm{~min}$ at 13 , $200 \mathrm{rpm}$. Separation of the reaction products was performed with a Merck $4.6 \times 50 \mathrm{~mm}$ Chromolith ${ }^{\circledR}$ SpeedROD RP-18e column with $\mathrm{ACN} / \mathrm{H}_{2} \mathrm{O}(60: 40, v / v)$ as the eluent.

\section{Results}

Comparative in silico analysis of $\mathrm{crtC}$ genes The $\mathrm{Rg}$-crtC nucleotide sequence was subjected to a BLAST search in order to identify sequence similarity in different databases. One hundred forty-five hits were identified, of which 119 were representatives of Proteobacteria. BLASTing the protein sequence of $\mathrm{RgCrtC}$ resulted in $100 \mathrm{CrtC}(-\mathrm{like})$ sequences, which were aligned in order to investigate if there are any conserved group clusters present (see Electronic Supplementary Material Fig. S1). Indeed, they showed highly conserved regions (Fig. 2, for simplification, the seven sequences used in this study were selected for visualization) distributed along the sequence ranging from amino acid residues $\sim 170$ to $\sim 405$ ( $R g$ numbering). Interestingly, the Nterminal part of the sequence does not contain any conserved amino acids and shows a lot of variation in length, indicating that this region is probably not necessary for $\mathrm{CrtC}$ activity. Additionally, we found some blocks of highly conserved residues throughout the $\mathrm{C}$-terminal part of the genes and a cluster of totally conserved residues in the middle of the genes (amino acids $240-280$ in $R g$ numbering).

Residues involved in the catalysis tend to be highly conserved in a set of homologous proteins that exhibit the same reaction. On the other hand, sequence insertion and sections of low sequence similarity tend to occur in the less important loop regions (Zvelebil et al. 1987). The conserved blocks in $\mathrm{CrtC}$ homologues could indicate that these regions contain the amino acid residues most important for the hydratase activity, specifically those involved in catalysis and substrate binding. 


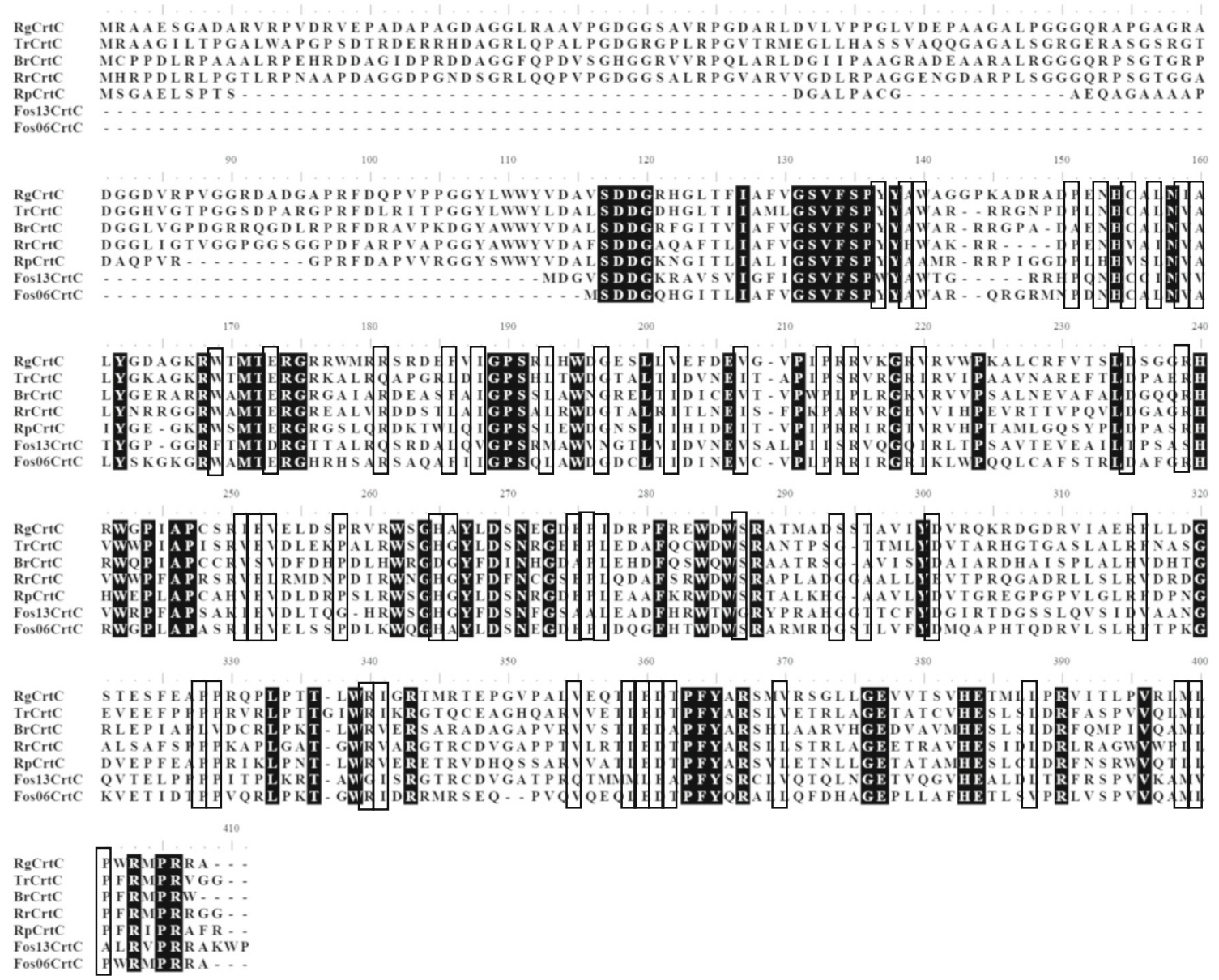

Fig. 2 Multiple sequence alignment of CrtC protein sequences probed in this study showing conserved amino acids. Identical amino acids are highlighted in black. Positions with only two different amino acids are surrounded by boxes

From a catalytic point of view, specific amino acids are commonly involved as active residues in acid-base-type catalyzed reactions in the active sites of enzymes (Bartlett et al. 2002). The amino acids aspartic acid (D) or glutamic acid (E) are usually the catalytic acid or base, while tyrosine (Y), tryptophan $(\mathrm{W})$, and histidine $(\mathrm{H})$ typically function as the other part of the charge relay pair (Puthan Veetil et al. 2012). These amino acids are, therefore, the most probable candidates for the catalytic hydration. Four Trp residues, three Tyr residues, and one of each His and Asp residues are fully conserved over the $100 \mathrm{CrtCs}$ (Supplemental Fig. S1). The totally conserved Asp268 (Rg numbering) seems to be the most probable candidate for the acid catalysis since it is situated in the middle of the highly conserved region. Furthermore, a fully conserved Tyr residue and a His residue are within close distance, which is important for the contact with the substrate. Fully conserved His and Trp residues are approximately 30 amino acids away but could be in close proximity upon folding of the protein.

Production of recombinant wild type and mutant CrtCs and enzymatic activity Six potential $\mathrm{CrtCs}$ were selected for expression and activity studies based on sequence identity with $\mathrm{RgCrtC}$ and availability of the corresponding gene constructs. They originate from all three Proteobacteria subclasses (Alphaproteobacteria, Betaproteobacteria, and Gammaproteobacteria) with two additional constructs originating from metagenomic samples from the Delaware River (USA). Figure 3 displays the phylogenetic analysis constructed with protein sequences of the selected $\mathrm{CrtC}$ homologues. $\operatorname{Tr} \mathrm{CrtC}$ shows the closest relationship to $\mathrm{RgCrtC}$, followed by $\mathrm{BrCrtC}$ (55 and $47 \%$ sequence identity, respectively). The combined results of Pfam search and Conserved Domain Database search showed that all seven CrtCs belong to the PF07143 family consisting of several purple photosynthetic bacterial hydroxyneurosporene synthase $(\mathrm{CrtC})$ proteins. Six out of the seven selected CrtCs could be overexpressed from pET15b in E. coli (Fig. 4). Bands with apparent molecular weight of $32 \mathrm{kDa}(\mathrm{Fos} 13 \mathrm{CrtC}), 38 \mathrm{kDa}(R p \mathrm{CrtC})$, and $44 \mathrm{kDa}(\operatorname{Rr} \mathrm{CrtC}, \mathrm{Br} \mathrm{CrtC}, \operatorname{Tr} \mathrm{CrtC}$, and $\operatorname{RgCrtC})$ were visualized on SDS-PAGE and were consistent with the values calculated from the deduced amino acid sequences. $\operatorname{Tr} \mathrm{CrtC}$ shows two protein bands of 44 and $38 \mathrm{kDa}$ as seen before 


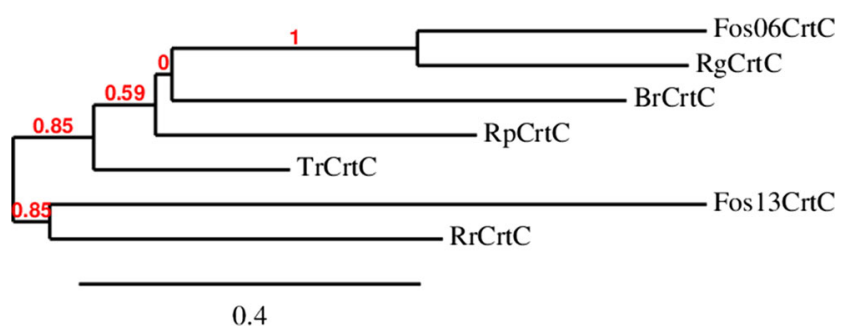

Fig. 3 Rooted phylogenetic tree showing the evolutionary relationship between the selected carotenoid 1,2-hydratases. The scale bar represents 0.4 substitutions per amino acid site. TrCrtC Thiocapsa roseopersicina (GI 31621263), BrCrtC Bradyrhizobium sp. BTAi1 (GI 146403799), Fos06CrtC uncultured Proteobacterium DelRiverFos06H03 (GI 61653228), Fos 13CrtC uncultured Proteobacterium DelRiverFos13D03 (GI 61653190), Rp CrtC Rhodopseudomonas palustris (GI 115515977), RrCrtC Rhodospirillum rubrum (GI 83574254), RgCrtC Rubrivivax gelatinosus (GI 29893477)

(Hiseni et al. 2011). No expression band could be identified for Fos06CrtC. Although relatively good expression was achieved for most of the CrtCs, only two were active with lycopene as the substrate in the standard enzymatic assay, i.e., $\operatorname{RgCrtC}$ and $\operatorname{Tr} \mathrm{CrtC}$ (data not shown).

The amino acid residues that might be important for catalysis (vide supra) were substituted by the amino acid alanine in both active enzymes. In addition, truncated ( $\operatorname{Tr} \mathrm{CrtC}$ and $R g C r t C)$ and N-terminal point mutants ( $T r C r t C)$ were constructed and analyzed to confirm the importance of the $\mathrm{N}$ terminal part of $\mathrm{CrtC}$ for the catalytic activity. The activity of the truncated versions was fully retained. Despite the still unknown reason for the truncation, we were able to identify the cleavage site between S57 and S58 by MS analysis. In order to exclude that this truncation only takes place in recombinant expression, the S58 position was modified by substitutions with valine or glutamine (see Electronic Supplementary Materials, including Fig. S2).

All mutants (Table 2) were successfully cloned and expressed in E. coli BL21 (Fig. 5a). However, clear differences in expression levels were observed. While the removal of the N-terminus resulted in an increased expression level, all point mutations negatively influenced the expression of the protein. In order to ensure that $\mathrm{CrtC}$ was present, all mutants were purified from the membrane fraction. As can be seen in Fig. 5b, all mutants could be purified by chromatography over a Ni column binding the His tag and showed a band at 38 or $44 \mathrm{kDa}$, which was absent in the negative control sample (pET15b). In the case of $T r \mathrm{CrtC}$ constructs, only very weak protein bands are detected after the purification (Fig. 5b, lanes 8 and 10-15). The purification usually has to be performed as soon as it is expressed (before the cleavage of the $\mathrm{N}$ terminal part including the His tag), which was not the case here.

Next to the analysis of the expression levels, the activities of all constructed mutants were measured with lycopene as the substrate (Fig. 6). As the expression and purification levels were very low for some of the mutants and the activity of $\mathrm{CrtC}$, in general, is very low, crude extracts were used for the activity assays. Consequently, the results cannot be quantitatively compared. However, in combination with the expression levels as shown in Fig. 5a, indicative conclusions can be drawn. When looking at the results from the alanine mutants, it appears that four key residues were identified, which have a potentially important role in the hydration mechanism. By replacing each of the amino acids H239, W241, Y266, and D268 individually by an alanine in $\mathrm{RgCrtC}$, the activity is completely destroyed. The same mutations of the corresponding amino acids in $\operatorname{Tr} \mathrm{CrtC}$, i.e., H237, W239, and D266, also resulted in CrtC inactivation. Unfortunately, the mutagenesis of Y264 in $\operatorname{Tr} \mathrm{CrtC}$ was not successful and, therefore, could not be included in this study. However, based on all the results, one could expect that the mutation of Y264 in $\operatorname{TrCrtC}$ would lead to inactivation, as seen in $\mathrm{RgCrtC}$. On the other hand, the less conserved $\mathrm{H} 264$ in $\mathrm{RgCrtC}$ and the corresponding histidine residue in $\operatorname{Tr} \mathrm{CrtC}$ (H262) seem not to have any functional role. The mutants fully retained activity and

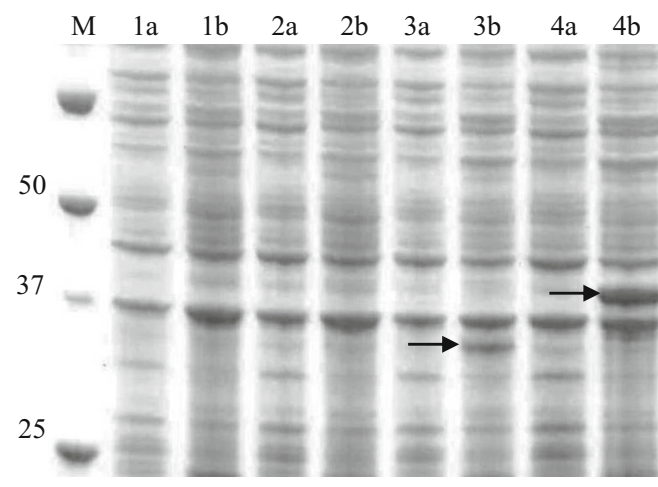

Fig. 4 SDS-PAGE (10\%) analysis of CrtC expression in E. coli BL21. $M$ precision plus protein standard. The first lane $(a)$ of each sample shows cells before induction with $0.6 \mathrm{mM}$ IPTG, and the second lane $(b)$ shows cells after $4 \mathrm{~h}$ of expression at $37^{\circ} \mathrm{C} .1$ pET15b control, 2 Fos06CrtC

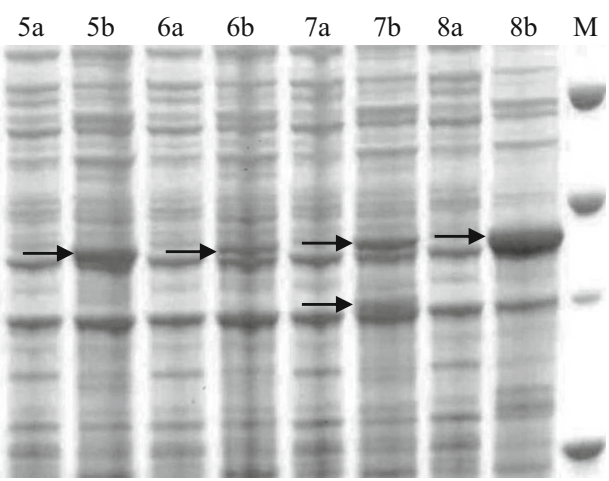

(32 kDa), 3 Fos13CrtC (32 kDa), 4 RpCrtC (38 kDa), $5 \operatorname{RrCrtC}(44 \mathrm{kDa})$, $6 \operatorname{BrCrtC}(44 \mathrm{kDa}), 7 \operatorname{Tr} \mathrm{CrtC}(44 \mathrm{kDa}), 8 \operatorname{RgCrtC}(44 \mathrm{kDa})$. The indicated molecular weights are deduced from amino acid sequences. $\mathrm{CrtC}$ expression bands are indicated by arrows 

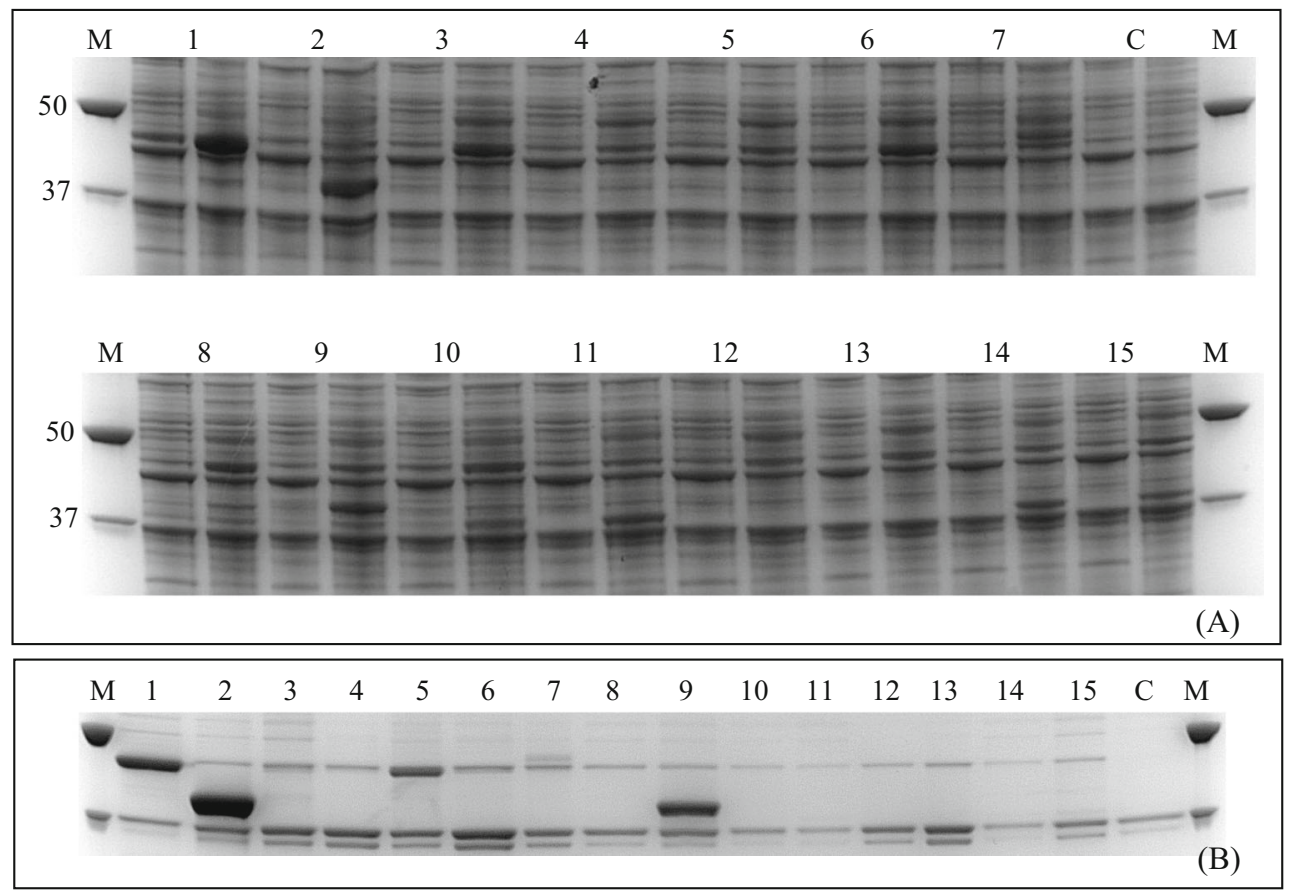

Fig. 5 SDS-PAGE (10\%) analysis of expression (a) and IMAC purification from membrane (b) of $\mathrm{CrtCs}$ from $R$. gelatinosus ( $\mathrm{RgCrtC}$ ) (lanes 1-7) and T. roseopersicina ( $\operatorname{Tr} \mathrm{CrtC})$ (lanes 8-15) wild type and mutants. $M$ precision plus protein standard. $C$ pET15b control. a The first lane of each sample shows cells before induction with $0.1 \mathrm{mM} \mathrm{IPTG}$, and

even showed slightly increased activity when the expression levels were considered. For instance, the truncated $\operatorname{Tr} \mathrm{CrtC}$ and H262A mutant showed almost the same level of expression (Fig. 5a, lanes 9 and 14) but the activity of H262A mutant was 1.3-fold higher (Fig. 6). The same was observed for $\mathrm{RgCrtC}$, where the expression of the wild type is much higher than that

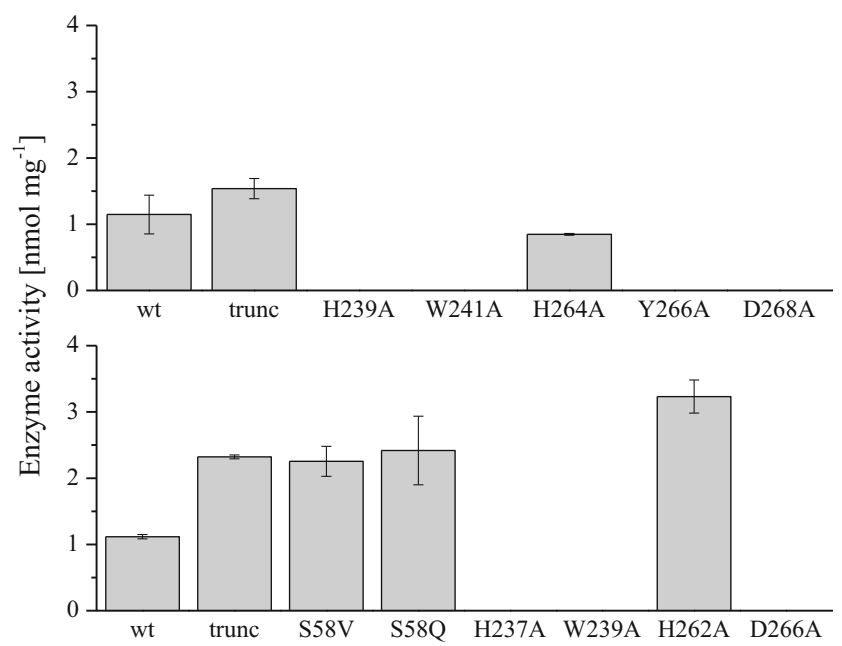

Fig. 6 Enzymatic activity of wild type $(w t)$ and mutant $\mathrm{CrtC}$ from $R$. gelatinosus (upper) and T. roseopersicina (lower). Extracts from $E$. coli cells expressing the respective enzymes were assayed with $20 \mu \mathrm{M}$ lycopene in $50 \mathrm{mM} \mathrm{Na}_{2} \mathrm{HPO}_{4}$ sodium phosphate $(\mathrm{pH} 8.0)$ at $28{ }^{\circ} \mathrm{C}$ overnight. trunc variants with missing $\mathrm{N}$-terminal residues $1-45$ $(\mathrm{RgCrtC})$ and $1-57(\operatorname{Tr} \mathrm{CrtC})$ the second lane shows cells after overnight expression at $25^{\circ} \mathrm{C} .1 \mathrm{RgCrtC}$ wild type, $2 R g C r t C$ truncated, $3 R g C r+C ~ H 239 A, 4 R g C r t C$ W241A, 5 $R g C r t C$ H264A, 6 RgCrtC Y266A, 7 RgCrtC D268A, $8 \operatorname{TrCrtC}$ wild type, $9 \operatorname{Tr} \mathrm{CrtC}$ truncated, $10 \operatorname{Tr} \mathrm{CrtC} \mathrm{S} 58 \mathrm{~V}, 11 \operatorname{Tr} \mathrm{CrtC}$ S58Q, $12 \operatorname{Tr} \mathrm{CrtC}$ H237A, $13 \operatorname{Tr}$ CrtC W239A, $14 \operatorname{Tr}$ CrtC H262A, $15 \operatorname{Tr}$ CrtC D266A

of the mutant H264A, but both showed approximately the same activity.

\section{Discussion}

The main purpose of this study was to get more insight into the hydration mechanism of carotenoid 1,2-hydratases. First, the distribution of these enzymes was assessed by subjecting the $R g$-crtC nucleotide sequence to a BLAST search. Although $R$. gelatinosus belongs to the Betaproteobacteria, more than $69 \%$ of the identified 119 hits were from Alphaproteobacteria and only $11 \%$ from Betaproteobacteria. Similarly, Igarashi et al. (2001) observed that most of the photosynthesis gene products from $R$. gelatinosus showed high sequence identities to the gene products of $R$. palustris, an Alphaproteobacteria member. They explain this occurrence as horizontal transfer of the photosynthesis gene clusters from an ancestral species belonging to the Alphaproteobacteria to that of the Betaproteobacteria, which might also have happened to the $\mathrm{CrtC}$ genes. The identified $\mathrm{CrtC}$ sequences were aligned in order to discover conserved group clusters. In total, 33 amino acids were found to be fully conserved. No conserved residues were identified in the $\mathrm{N}$-terminal part of the sequence (amino acids 1-125 in $R g$ numbering), which is in agreement with our earlier hypothesis that this region is probably not necessary for CrtC activity (Hiseni et al. 2011). This was also confirmed by 
Fig. 7 Enzyme-catalyzed cyclisation of squalene to hopene and hopanol

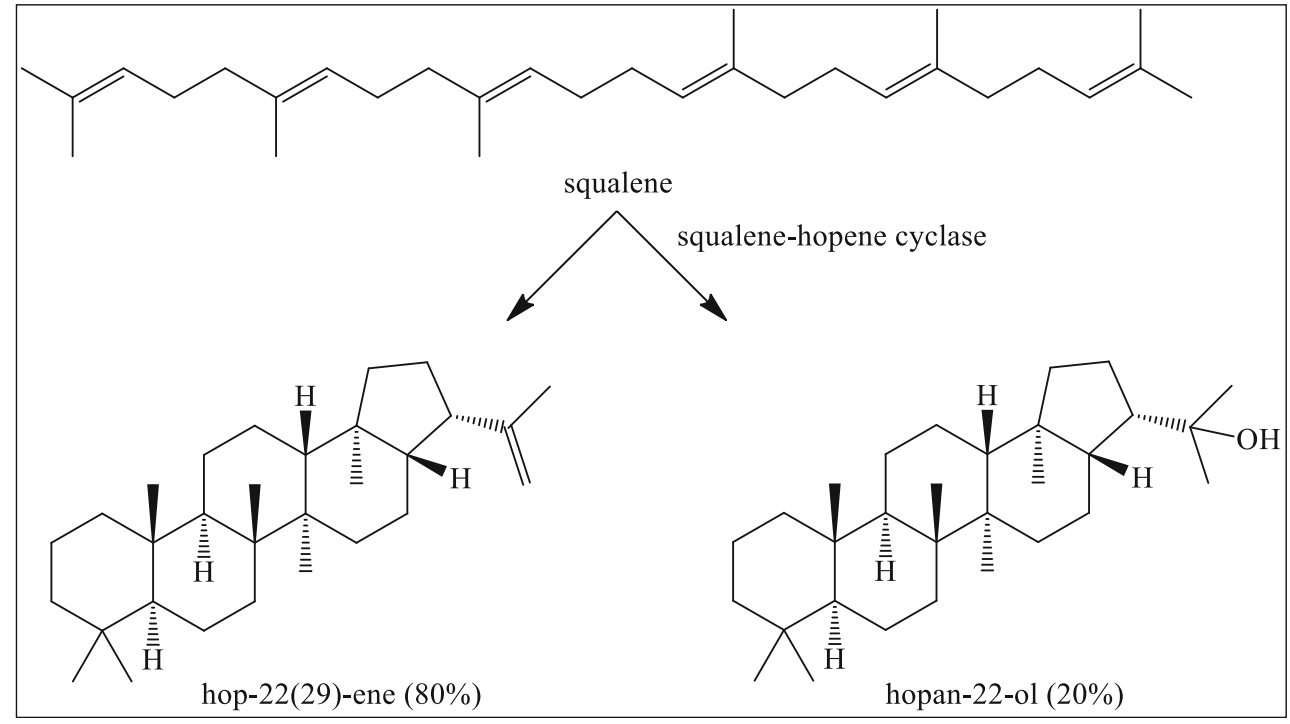

the activity of the truncated variants. Furthermore, this also accounts for the absence of this part and, thus, the shorter DNA sequences for many CrtCs, including Fos06, Fos13, and partly $R p C r t C$, when compared to $R g C r t C$ (Fig. 2, Supplemental Fig. S1). This part is, hence, not involved in the catalytic mechanism.

Six out of the seven selected CrtCs could be overexpressed in E. coli (Fig. 4). The fact that all CrtCs share highly conserved regions in the amino acid sequence indicates that they are performing the same or similar biochemistry. However, no activity whatsoever could be detected for four out of six CrtCs in the standard lycopene hydration assay. At this point, it is unclear whether this is due to reasons of low activity in the cell extract and/or substrate specificity. Since CrtCs are active in different parts of the carotenoid pathway, substrate specificities can differ depending on the carotenoid produced by the organism where the enzyme originated (Kovács et al. 2003).

We have stated earlier that the hydration can take place through an acid-base-type catalysis, resulting in the identification of five possible key residues. The identified key residues H239, W241, Y266, and D268 in RgCrtC and the corresponding residues in $\operatorname{Tr} \mathrm{CrtC}$ were probed by generating alanine point mutants thereof. The absence of activity upon individual substitution leads to the hypothesis that they are involved in the hydration catalysis. Furthermore, they are all in close distance to each other on the protein chain (Fig. 2). The fact that there is only one other residue between $\mathrm{H} 239$ and W241 or Y266 and D268 is consistent with residues in space pointing in the same direction in a beta strand or loop (one residue pointing upward, the next downward). In order to investigate how these newly identified key residues could be involved in the catalytic hydration reaction, we built a $3 \mathrm{D}$ structure of $\operatorname{Tr} \mathrm{CrtC}$ by homology modeling. The closest known 3D structure, the putative AttH protein from Nitrosomonas europaea, showed only a sequence identity of
$17 \%$ to the CrtC (see Electronic Supplementary Material Figs. S3, S4, and S5). This is not enough for a reliable model. However, this model does indicate the possibility of the presence of such an active site.

These four residues, which are conserved throughout the whole CrtC family, are also found in the active site of squalene-hopene cyclase (SHC) (Wendt et al. 2000). SHC catalyzes the cyclization reaction of squalene to hopene as a major product (Fig. 7). Hopanol is also formed to a minor extent. The proposed mechanism for cyclases is proton-triggered polycyclization, whereby the intermediate carbocation is stabilized by aromatic amino acids. Next to the stabilization role of the aromatic amino acids, they also create a hydrophobic environment in order to prevent quenching of the cation by water. The cyclization cascade is terminated by a well-positioned enzymatic base. The formation of the alcohol side product suggests significant water accessibility at the termination region of the active site. The acidic residue aspartate (D376), which

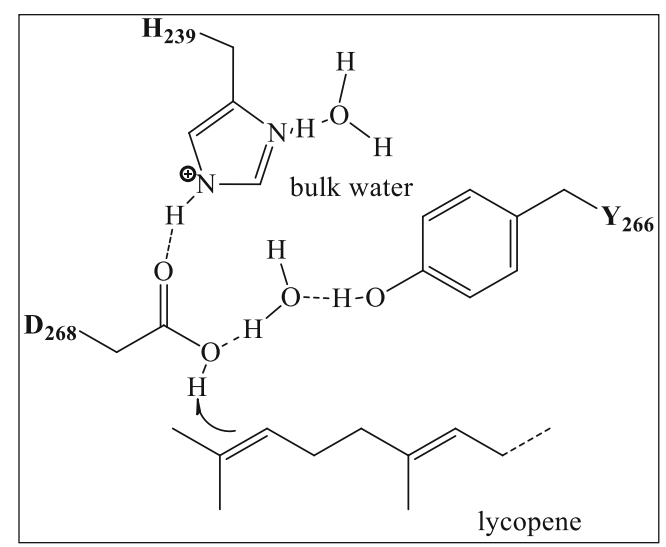

Fig. 8 Proposed mechanism for the initial protonation during lycopene hydration 
is located in the center of the active site in SHC, is the likely general acid responsible for protonating the $\mathrm{C} 3$ atom of the squalene substrate (Wendt et al. 2000). The acidity of D376 is enhanced by a connection to the side chain of Y495 through a water molecule. The tyrosine positions the proton on aspartic acid into the anti-orientation, turning it more acidic. Carboxylic acid protons in anti-orientation have been estimated to be $10^{4}$ times more acidic than the biologically more relevant syn-oriented protons (Gandour 1981).

Because of the probable similarity of the initial protonation reactions of squalene and lycopene, we assume that the residues involved in catalysis will be alike in SHC and CrtC. Therefore, we propose the following mechanism for $R g C r t C$. D268 is the catalytic acid that initiates the hydration of lycopene (Fig. 8). Upon diffusion of lycopene into the active site, the $\mathrm{C} 2$ atom of the substrate needs to be positioned near the proton of D268 that putatively will be added onto the substrate. In order to enhance the acidity of the catalytic D268 for olefin protonation, the amino acid is directly bonded to H239 and to Y266 through an ordered water molecule, similar to what has been proposed for SHC (Wendt et al. 2000). This hypothesis is supported by the results that mutation of one of these three amino acids leads to inactivation of enzymatic activity. In contrast to SHC, where premature quenching of the cationic intermediate by water or nucleophiles is prevented by well-positioned aromatic amino acids, a water molecule is added to lycopene to yield the desired hydroxylated lycopene derivative. This suggests that the active site of $\mathrm{CrtC}$ has more water molecules present, so that the interaction between the substrate and solvent water molecules is more significant. The aromatic amino acid Trp266 might be involved in the correct positioning of the hydrophobic substrate and the stabilization of the intermediate carbocation.

In conclusion, overall results indicate that residues H239, W241, Y266, and D268 in RgCrtC are probably involved in an acid-base-type hydration. The absence of activity upon individual substitution of these residues by an alanine agrees with a role in the catalytic cycle. We hypothesize that they are involved in the initial protonation, which would be followed by quenching of the carbocation by a water molecule, resulting in the hydration product. From our findings, it becomes clear that the complete structure of a $\mathrm{CrtC}$, through crystallization studies, will be pivotal to really unravel the mechanism for this intriguing enzyme. Nevertheless, the results of this study produce for the first time a workable hypothesis for the catalytic mechanism of carotenoid 1,2hydratase and open the field for the engineering of this enzyme towards industrially relevant mutants.
Acknowledgments We thank Dr. Jaap Jongejan (Delft University of Technology) for his advice on finding the potentially important residues in carotenoid 1,2-hydratases.

Compliance with ethical standards This project was financially supported by The Netherlands Ministry of Economic Affairs and the B-Basic partner organizations (http://www.b-basic.nl) through BBasic, a public-private NWO-ACTS programme [Advanced Chemical Technologies for Sustainability (ACTS)].

Conflict of interest The authors declare that they have no competing interests.

Open Access This article is distributed under the terms of the Creative Commons Attribution 4.0 International License (http:// creativecommons.org/licenses/by/4.0/), which permits unrestricted use, distribution, and reproduction in any medium, provided you give appropriate credit to the original author(s) and the source, provide a link to the Creative Commons license, and indicate if changes were made.

\section{References}

Altschul SF, Gish W, Miller W, Myers EW, Lipman DJ (1990) Basic local alignment search tool. J Mol Biol 215(3):403-410. doi:10.1016/ S0022-2836(05)80360-2

Armstrong GA (1997) Genetics of eubacterial carotenoid biosynthesis: a colorful tale. Annu Rev Microbiol 51(1):629-659. doi:10.1146/ annurev.micro.51.1.629

Armstrong GA, Hearst JE (1996) Carotenoids 2: genetics and molecular biology of carotenoid pigment biosynthesis. FASEB J 10(2):228237

Bartlett GJ, Porter CT, Borkakoti N, Thornton JM (2002) Analysis of catalytic residues in enzyme active sites. J Mol Biol 324(1):105121. doi:10.1016/S0022-2836(02)01036-7

Cazzonelli CI (2011) Carotenoids in nature: insights from plants and beyond. Funct Plant Biol 38(11):833-847. doi:10.1071/FP11192

Dereeper A, Audic S, Claverie J-M, Blanc G (2010) BLASTEXPLORER helps you building datasets for phylogenetic analysis. BMC Evol Biol 10(1):1-6. doi:10.1186/1471-2148-10-8

Dereeper A, Guignon V, Blanc G, Audic S, Buffet S, Chevenet F, Dufayard J-F, Guindon S, Lefort V, Lescot M, Claverie J-M, Gascuel O (2008) Phylogeny.fr: robust phylogenetic analysis for the non-specialist. Nucleic Acids Res 36(suppl 2):W465-W469. doi:10.1093/nar/gkn180

Evans CM, Kirby AJ (1984) A model for olefin hydration: intramolecular nucleophilic addition of phenolate oxygen to the unactivated double bond. J Chem Soc Perkin Trans 2(7):1259-1267. doi:10.1039/ P29840001259

Finn RD, Mistry J, Tate J, Coggill P, Heger A, Pollington JE, Gavin OL, Gunasekaran P, Ceric G, Forslund K, Holm L, Sonnhammer ELL, Eddy SR, Bateman A (2010) The Pfam protein families database. Nucleic Acids Res 38(suppl 1):D211-D222. doi:10.1093/nar/ gkp985

Gandour RD (1981) On the importance of orientation in general base catalysis by carboxylate. Bioorg Chem 10(2):169-176. doi:10. 1016/0045-2068(81)90020-1

Giraud E, Hannibal L, Fardoux J, Jaubert M, Jourand P, Dreyfus B, Sturgis JN, Verméglio A (2004) Two distinct crt gene clusters for two different functional classes of carotenoid in Bradyrhizobium. J Biol Chem 279(15):15076-15083. doi:10.1074/jbc.M312113200

Hammer SC, Syrén P-O, Seitz M, Nestl BM, Hauer B (2013) Squalene hopene cyclases: highly promiscuous and evolvable catalysts for 
stereoselective CC and CX bond formation. Curr Opin Chem Biol 17(2):293-300. doi:10.1016/j.cbpa.2013.01.016

Hiseni A, Arends IWCE, Otten LG (2011) Biochemical characterization of the carotenoid 1,2-hydratases (CrtC) from Rubrivivax gelatinosus and Thiocapsa roseopersicina. Appl Microbiol Biotechnol 91(4): 1029-1036. doi:10.1007/s00253-011-3324-1

Hiseni A, Arends IWCE, Otten LG (2015) New cofactor-independent hydration biocatalysts: structural, biochemical, and biocatalytic characteristics of carotenoid and oleate hydratases. ChemCatChem 7(1):29-37. doi:10.1002/cctc.201402511

Igarashi N, Harada J, Nagashima S, Matsuura K, Shimada K, Nagashima KVP (2001) Horizontal transfer of the photosynthesis gene cluster and operon rearrangement in purple bacteria. J Mol Evol 52(4):333341. doi:10.1007/s002390010163

Jensen SL, Cohen-Bazire G, Stanier RY (1961) Biosynthesis of carotenoids in purple bacteria: a re-evaluation based on considerations of chemical structure. Nature 192(4808):1168-1172. doi:10.1038/ $1921168 \mathrm{a} 0$

Jin J, Hanefeld U (2011) The selective addition of water to $\mathrm{C}=\mathrm{C}$ bonds; enzymes are the best chemists. Chem Commun 47(9):2502-2510. doi: $10.1039 / \mathrm{C0CC} 04153 \mathrm{~J}$

Kammann M, Laufs J, Schell J, Gronenbom B (1989) Rapid insertional mutagenesis of DNA by polymerase chain reaction (PCR). Nucleic Acids Res 17(13):5404. doi:10.1093/nar/17.13.5404

Kourist R, Domínguez de María P, Bornscheuer UT (2008) Enzymatic synthesis of optically active tertiary alcohols: expanding the biocatalysis toolbox. ChemBioChem 9(4):491-498. doi:10.1002/cbic. 200700688

Kovács ÁT, Rákhely G, Kovács KL (2003) Genes involved in the biosynthesis of photosynthetic pigments in the purple sulfur photosynthetic bacterium Thiocapsa roseopersicina. Appl Environ Microbiol 69(6):3093-3102. doi:10.1128/aem.69.6.3093-3102.2003

Landt O, Grunert H-P, Hahn U (1990) A general method for rapid sitedirected mutagenesis using the polymerase chain reaction. Gene 96(1):125-128. doi:10.1016/0378-1119(90)90351-q

Larkin MA, Blackshields G, Brown NP, Chenna R, McGettigan PA, McWilliam H, Valentin F, Wallace IM, Wilm A, Lopez R, Thompson JD, Gibson TJ, Higgins DG (2007) Clustal W and Clustal X version 2.0. Bioinformatics 23:2947-2948. doi:10.1093/ bioinformatics/btm 404

Marchler-Bauer A, Lu S, Anderson JB, Chitsaz F, Derbyshire MK, DeWeese-Scott C, Fong JH, Geer LY, Geer RC, Gonzales NR,
Gwadz M, Hurwitz DI, Jackson JD, Ke Z, Lanczycki CJ, Lu F, Marchler GH, Mullokandov M, Omelchenko MV, Robertson CL, Song JS, Thanki N, Yamashita RA, Zhang D, Zhang N, Zheng C, Bryant SH (2011) CDD: a conserved domain database for the functional annotation of proteins. Nucleic Acids Res 39(Suppl 1):D225D229. doi:10.1093/nar/gkq1189

Patel NJ, Britton G, Goodwin TW (1983) Use of deuterium labelling from deuterium oxide to demonstrate carotenoid transformations in photosynthetic bacteria. Biochim Biophys Acta, Gen Subj 760(1): 92-96. doi:10.1016/0304-4165(83)90128-9

Puthan Veetil V, Fibriansah G, Raj H, Thunnissen A-MWH, Poelarends GJ (2012) Aspartase/fumarase superfamily: a common catalytic strategy involving general base-catalyzed formation of a highly stabilized aci-carboxylate intermediate. Biochemistry 51(21):42374243. doi:10.1021/bi300430j

Sarkar G, Sommer SS (1990) The 'megaprimer' method of site-directed mutagenesis. BioTechniques 8(4):404-407

Sun Z, Shen S, Wang C, Wang H, Hu Y, Jiao J, Ma T, Tian B, Hua Y (2009) A novel carotenoid 1,2-hydratase (CruF) from two species of the non-photosynthetic bacterium Deinococcus. Microbiology 155(8):2775-2783. doi:10.1099/mic.0.027623-0

Umeno D, Tobias AV, Arnold FH (2005) Diversifying carotenoid biosynthetic pathways by directed evolution. Microbiol Mol Biol Rev 69(1):51-78. doi:10.1128/mmbr.69.1.51-78.2005

Waidner LA, Kirchman DL (2005) Aerobic anoxygenic photosynthesis genes and operons in uncultured bacteria in the Delaware River. Environ Microbiol 7(12):1896-1908. doi:10.1111/j.1462-2920. 2005.00883.x

Wendt KU, Schulz GE, Corey EJ, Liu DR (2000) Enzyme mechanisms for polycyclic triterpene formation. Angew Chem Int Ed 39(16): 2812-2833. doi:10.1002/1521-3773(20000818)39:16<2812::AIDANIE2812>3.0.CO;2-\#

Yeliseev AA, Kaplan S (1997) Anaerobic carotenoid biosynthesis in Rhodobacter sphaeroides 2.4.1: $\mathrm{H}_{2} \mathrm{O}$ is a source of oxygen for the 1-methoxy group of spheroidene but not for the 2-oxo group of spheroidenone. FEBS Lett 403(1):10-14. doi:10.1016/S00145793(97)00006-9

Zvelebil MJ, Barton GJ, Taylor WR, Sternberg MJE (1987) Prediction of protein secondary structure and active sites using the alignment of homologous sequences. J Mol Biol 195(4):957-961. doi:10.1016/ 0022-2836(87)90501-8 